\title{
Bright Light Therapy's Effect on Night Shift Health Care Workers Who Suffer from Light Induced Misalignment of Circadian Rhythms
}

\author{
Mackenzie Peed ${ }^{1}$ and John Murnan ${ }^{1}$ \\ ${ }^{1}$ Etowah High School, Woodstock, GA, USA

\section{$\underline{\text { ABSTRACT }}$}

Modern-day society is based on a high pace lifestyle that people are constantly attempting to keep up with. The invention of the light completely changed society, allowing people to work outside of daylight hours. As countries become more and more industrialized, the need for 24/7 productivity becomes more and more common. From transportation to mining to medicine, individuals willing to work unconventional hours are necessary for the sustainability of the industry. This group of people, working outside "normal" work hours such as between 7 a.m. and 6 p.m. or shifts longer than eight hours, are known as shift workers. They make up about 26.5 million people in the employed population in the United States and about 100 million around the world (Moore-Ede \& Platika, 2018). Shift work affects a person's circadian rhythm, causing issues. "Circadian rhythm mainly controls the daily wake and sleep cycle and regulates physiological processes including hormone secretion, body temperature, feeding behavior, cell cycle progression, and drug, glucose, and xenobiotic metabolism" (Khan et al., 2018, p.2). This circadian rhythm is controlled by the circadian clock and is regulated through clock genes. Light exposure greatly influences circadian rhythms and specifically affects the release of the hormone melatonin which activates specific receptors MTI and MT2 that mediate sleep-promoting effects.

\section{Introduction}

Modern-day society is based on a high pace lifestyle that people are constantly attempting to keep up with. The invention of the light completely changed society, allowing people to work outside of daylight hours. As countries become more and more industrialized, the need for $24 / 7$ productivity becomes more and more common. From transportation to mining to medicine, individuals willing to work unconventional hours are necessary for the sustainability of the industry.

This group of people, working outside "normal" work hours such as between 7 a.m. and 6 p.m. or shifts longer than eight hours, are known as shift workers. They make up about 26.5 million people in the employed population in the United States and about 100 million around the world (Moore-Ede \& Platika, 2018). Shift work affects a person's circadian rhythm, causing issues. "Circadian rhythm mainly controls the daily wake and sleep cycle and regulates physiological processes including hormone secretion, body temperature, feeding behavior, cell cycle progression, and drug, glucose, and xenobiotic metabolism" (Khan et al., 2018, p.2). This circadian rhythm is controlled by the circadian clock and is regulated through clock genes. Light exposure greatly influences circadian rhythms and specifically affects the release of the hormone melatonin which activates specific receptors MTI and MT2 that mediate sleep-promoting effects. When our circadian rhythm is disrupted due to light and dark disturbances this is known as circadian misalignment which causes melatonin and clock genes to be disrupted, causing health implications.

Various studies have been conducted showing the correlation between this circadian misalignment and sleep disorders, extreme fatigue, metabolic disruption, and even carcinogenic effects with long term exposure, but the mechanisms that contribute to circadian misalignment and these health implications is not fully understood. One mechanism 
that has been investigated is the use of melatonin rhythm as a marker for the human circadian timing rhythm, which Shanahan \& Zeitzer (1997) found is one of the only circadian markers with a well-defined link with the nervous system. I will further this research by applying this mechanism of melatonin rhythm and specifically applying it to health care workers. These shift workers suffer from severe circadian misalignment and need to be further researched to provide a solution.

\section{Literature Review}

\section{Light's Effect on Circadian Rhythms}

Improper light exposure causes circadian misalignment due to the wavelengths present in this light. Moore-Ede $\&$ Platika, researchers helping to develop and distribute new lighting systems through the establishment of Circadian ZircLight Inc, found that there are special photoreceptor cells in the retina containing melanopsin, which is a pigment that has a blue light sensitivity in the 440-470nm range; this range is the visible light wavelength spectrum which causes melatonin suppression due to melanopsin sensitivity at night. These receptors send signals to the suprachiasmatic nucleus ( $\mathrm{SCN}$ ), a region of the hypothalamus, which governs the timing of melatonin production and releases into circulation through the pineal gland. This mechanism that controls melatonin rhythm is the most highly used marker for circadian timing rhythm. When these receptors are exposed to blue light in the morning, it causes peak productivity and is healthy, while blue light at night disrupts the circadian clock and causes a disruption cascade, disrupting hormones like melatonin and affecting clock genes. Since shift workers work hours outside of the normal work schedule, these individuals are highly affected by this harmful blue light during their night shift; this exposure can suppress melatonin over 80\% at night (MooreEde \& Platika, 2018). Lerman et al. (2012) also agrees that light exposure at night is harmful but states, "In experiments using notch filters in rodents, the effects of light on melatonin suppression, cortisol elevation, and circadian clock gene markers were found to be restricted to a narrow wavelength band between 470 and $480 \mathrm{~nm}$ " (p.246). This was also found in humans with a test showing that glasses cutting off wavelengths below $480 \mathrm{~nm}$ restricted these harmful effects while glasses cutting off at $460 \mathrm{~nm}$ did not. This shows a slight disagreement on whether light wavelength suppresses melatonin at a range of 440-470nm or 470-480.

\section{Health Risks Associated with Circadian Misalignment}

The harmful light wavelengths shift workers are exposed to during night shift causes circadian disruption through the disruption of circadian clock genes and melatonin suppression. Khan, Hou, Duan \& Yao, (2018), Boyce \& Hopwood (2013), Baron \& Reid (2014) all agree that this disruption of the circadian clock by light and dark disturbances causes health abnormalities. These health abnormalities include psychiatric disorders, sleep disorders, metabolic disruption, and carcinogenic effects. Mental and sleep disorders were shown through a study in rats, which found that, when exposed to light at a 6-hour phase advance each week, rats' survival rate dropped from $83 \%$ to $47 \%$ compared to those exposed to normal light (Khan et al., 2018). This drop-in survival rate can partially be explained through sleep deprivation caused by melatonin suppression. Since melatonin activates melatonin receptors which cause sleep onset, the MT1 and MT2, the absence of this melatonin would prevent this receptor activation and sleep would decline (Boyce \& Hopwood, 2013). Boyce \& Hopwood (2013) also connect this decline in melatonin secretion to mental illness stating that circadian misalignment can lead to depression due to changes in the normal circadian rhythm of core body temperature, cortisol secretion, noradrenaline levels, thyroid-stimulating hormone and melatonin secretion. Baron $\&$ Reid (2014) also found that a change in circadian clock proteins in the circadian pacemaker can cause manialike behavior in mice and there is evidence for weak associations between circadian clock gene polymorphisms, which are genes occurring in several different forms, and mood disorders. Clock gene polymorphisms are associated with SAD, seasonal affective disorder. PER3, BMAL1, and CLOCK, other clock genes, are associated with bipolar disorder, 
along with a stronger association with depressive symptoms. Long term exposure to this blue light at night is also associated with carcinogenic effects. Both Han-Xue (2019) and Lerman et al. (2012) agree that light at night is a probable carcinogen due to melatonin suppression. Since clock-controlled proteins influence the expression of between $50-80 \%$ of genes in mammals, the alteration of circadian clock genes through light exposure can partake in tumor development by altering the expression of other clock-controlled genes in cell cycle regulation, DNA damage repair, tumorigenesis and apoptosis, and tumor immunity. An increased risk of breast cancer in women of about 10$60 \%$ has been shown for those who work the night shift for more than 20 years (Han-Xue, 2019). Moore-Ede \& Platika, as discussed earlier, is working to sell a product to reduce these carcinogenic effects. Although this exposes his argument to potential bias, his findings do line up with that of Lerman and Han-Xue. His insights also elaborate on how melatonin suppression is carcinogenic, stating that melatonin has cancer-suppressing agents, therefore decreases in melatonin levels at night are correlated with increased breast cancer of 3258\% in women and a $200 \%$ increase in prostate cancer in men regularly exposed to this light (Moore-Ede \& Platika, 2018). These levels are higher than previously stated which may be due to the author's bias toward his product.

\section{Night Shift's Effect on Health Care Workers}

Circadian misalignment negatively affects any organization with workers working shift schedules due to its effect on health, safety, fatigue, and productivity. This is specifically seen in health care. Many individuals cannot adapt to light and dark disturbances due to hormone disruption and experience excessive sleepiness due to circadian rhythm's control on sleep drive. Lerman et al. (2012) argues that hormone disruption, caused by light and dark disturbances, affect individual's circadian rhythms which control sleep drive; sleep drive increases late at night and reaches peak productivity in the early morning. Individuals working night shift, therefore, experience severe fatigue while working due to circadian rhythm's effect on sleep drive. This fatigue has a negative impact on safety and performance within health care fields. Studies have shown that more fatigue-related incidents occur in the morning which is when peak sleepiness occurs due to circadian rhythms. Nurses who work more than 12.5-hour shifts are at a greater risk of suffering injury, making a medical error, and decreased vigilance; "The risk of an occupational sharps injury, of motor vehicle crash on the way home, or of making a serious medical error increases significantly because the hours on duty exceed 24 hours - compared with those working 16 hours - there are twice as many attentional failures and 36\% more serious medical errors" (Lerman et al., 2012, p.232). Moore-Ede \& Platika states that health costs along with accidents and lower productivity make night shift workers cost companies on average $\$ 10,000$ more than day shift workers; this is on average $\$ 900$ billion extra globally that night shift workers cost. Lerman et al. also shows a significant loss of money in health care due to shift work stating that a more recent study estimated a loss of $\$ 1967$ per employee per year in lost productivity due to sleep loss.

\section{Bright Light Therapy Exposure}

The technique of phase shifting has been discussed to accelerate the adaption to night shift work by shifting circadian rhythms. A model to demonstrate this phase shifting response is the phase response curve (PRC) which describes the direction and magnitude of phase shifts in the circadian clock (Lewy, 2010). The curve is made up of two opposite regions, phase delay and phase advance, and a dead zone (Figure 1). 


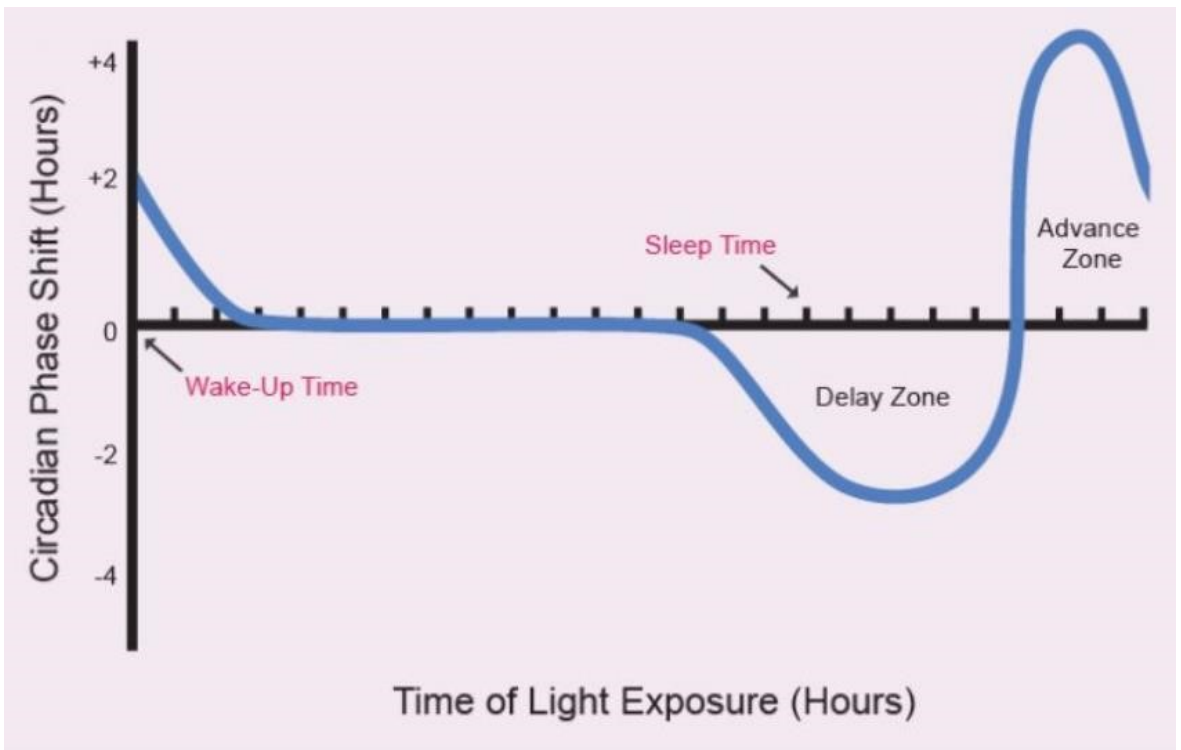

Figure 1. This is a phase response curve showing the delay and advance zones according to the time of light exposure and the magnitude of the phase shift. The dead zone would occur after the wake time and before the start of the delay zone.

The dead zone of the phase response curve in response to light occurs when there is little phase shifting which is during the biological day. The PRC curve has shown that bright light has phase shifting abilities, which are shown in the model's two opposite regions. When bright light exposure is administered early in the night a phase delay occurs, meaning circadian rhythms shift to a later time; this is shown in the phase delay zone. When light is administered later in the night it causes a phase advance, meaning circadian rhythms shift to an earlier time; this is shown by the phase advance zone. Circadian rhythms are aligned when melatonin peaks while sleeping, which for most, is late at night. Shift workers, however, sleep during the day, but their melatonin is still peaking at night when they are working. This bright light exposure at night can potentially cause a phase shift and alter melatonin peak levels so that they occur when shift workers are sleeping.

There are multiple ways to measure melatonin rhythms in relation to circadian timing. The best biomarker for the phase, or timing, of the circadian pacemaker is the dim light melatonin onset (DLMO), stated by Lewy, 2010. This is when the start of melatonin secretion occurs in dim ambient light conditions so suppression of melatonin by light is avoided, and it marks the beginning of one's biological night. Smith, Fogg, and Eastman (2009) along with Shanahan \& Zeitzer (1997) state that the DLMO occurs about 7 hours before the temperature minimum, which is an estimate for the sleepiest circadian time. A person's starting phase position, or their DLMO, will determine what time their temperature minimum occurs and when their sleepiest circadian time is. If the sleepiest circadian time occurs during one's night shift instead of sleep episode, then circadian rhythms are misaligned. Shanahan \& Zeitzer (1997) not only found when DLMO occurs, but also found a correlation between temperature minimum and peak melatonin levels. A study including 8 young men showed that the fitted maximum for plasma melatonin occurs on average about 1.9 hours before the fitted temperature minimum. The average temperature minimum occurs 1.4 hours before habitual wake time, in healthy young men. Therefore, if an individual's minimum temperature does not occur about 1.4 hours before their habitual wake time, melatonin is not peaking at the correct time and circadian rhythms are misaligned. While the DLMO is useful to determine when melatonin secretion starts, Shanahan \& Zeitzer's finding of when temperature minimum should occur in healthy individuals allows for the easiest measure of melatonin rhythm to assess circadian rhythms. 
Bright light therapy exposure has numerous benefits, but there are also risks. Lerman et al. (2012) agrees that bright light administered in the first half of the night delays the circadian clock and light administered in the second half advances the circadian clock, while also adding that light therapy can be used to accelerate adaption to night shift work by shifting circadian rhythms of alertness to increase performance. Therefore, bright light therapy can be used to address safety and performance issues. However, phase advance does not address health risks associated with blue light. As discussed earlier, bright light exposure at night of certain wavelengths can have negative impacts if proper filtration is not applied. Bright light therapy does allow for the phase shift of circadian rhythms which will promote sleep by allowing individuals to adapt to light and dark disturbances and therefore increase sleep drive. This will reduce fatigue in health care workers and increase safety and productivity. This will drastically change the lives of night shift health care workers by providing them with safe working conditions and an improved quality of life.

\section{Differences in Bright Light Therapy}

Bright light therapy is not a one fits all method. The timing of when bright light is administered determines whether a phase advance or delay occurs; either could be applied based on individual need. The intensity and duration of the light exposure determines how big the phase shift will be, along with how many consecutive days an individual is exposed. All these factors must be taken into consideration when attempting to realign an individual's circadian rhythms. A paper by Shanahan \& Zeitzer (1997), involved a study with individuals who were day workers, evening workers, and night workers. People who were day shift workers worked 7 a.m. to 3 p.m. and slept 10 p.m.-6 a.m.; this was used for baseline evaluation because these are normal working hours. Evening workers worked $3 \mathrm{pm}-11 \mathrm{pm}$ and slept 1am-9am, and night shift workers worked 11 p.m.-7 a.m. and slept 8 a.m.-4 p.m. They tested baseline conditions for two weeks then three weeks of night shift, three weeks of evening shift, and another three weeks of dayshift. The testing included watching when melatonin peeked and if this lined up with when the individual slept, as it should. The control group experienced no bright light therapy intervention, for they stayed in room light of 150 lux, and the treatment group was exposed to bright light of 5,000 lux during the entire night shift. Those receiving bright light treatment had melatonin rhythms that peaked during the scheduled day sleep episode, while those who stayed in room lighting failed to shift. A second study, by Smith, Fogg, and Eastman (2009), administered bright light therapy of 4100 lux to the experimental group during four, 15-minute bright light pulse intervals from light boxes containing fluorescent lamps.

Nineteen subjects underwent baseline evaluation for 15 days where they slept between $11 \mathrm{pm}$ and $7 \mathrm{am}$ and stayed in room lighting. Experimental subjects underwent eight-night shifts and worked from $11 \mathrm{pm}$ to $7 \mathrm{am}$. The first bright light pulse began at 12:45 AM and the last one ended at 4:00AM. The pulses were separated by 45 min of normal room light less than 50 lux. The results ended in a DLMO of about 3:00 am making the temperature minimum occur at $10 \mathrm{am}$. In this study, night shift workers, when on night shift, slept at 8:30 AM after night shifts and at 3:00 AM on days off. Therefore, a temperature minimum of about 10:00 AM puts the sleepiest circadian time early in the sleep period after night shifts and late in the sleep period on days off.

Shanahan \& Zeitzer's study achieved a more permanent phase shift, meaning that night shift worker's temperature minimum phase shifted to the middle of their daily sleep schedule at 12 p.m. compared to when it used to peak at 4 a.m. before bright light therapy. Smith, Fogg, and Eastman (2009) achieved a type of phase shift that caused melatonin peak to a comprisable time between day and night shift. Temperature minimum always occurred during a sleep episode but occurred either at the very beginning of the sleep episode when workers worked night shift and at the end of the sleep episode when workers had a day off. Both studies exhibited different intensities of light, different exposure times, and different numbers of executive days exposed to bright light therapy. All these differences determined how circadian rhythms reacted. 


\section{Conclusion}

This research shows how blue light disrupts circadian rhythms and the many health implications that can arise. These health abnormalities include psychiatric disorders, metabolic disruption, carcinogenic effects, sleep disorders, and extreme fatigue. Night shift health care workers are shown to be a large group of individuals at risk due to their working schedules. Extreme fatigue and sleep loss are shown to dramatically decrease worker productivity and increase medical errors. Bright light therapy has been shown to have phase shifting abilities according to the phase response curve and can accelerate adaption to night shift. By using temperature minimums to measure when melatonin reaches its peak level, circadian rhythms can be evaluated. This allows bright light therapy to alleviate safety and performance-related issues, but unless proper filtration is applied, health risks such as psychiatric disorders, metabolic disruption, and carcinogenic effects go unsolved. This research has addressed the use of melatonin rhythm as a marker for the human circadian timing rhythm, but research has yet to apply this to night shift health care workers. This gap in research needs to be further addressed and has led me to the research question, does light therapy cause a phase shift among night shift health care workers who suffer from light-induced misalignment of circadian rhythms? By using temperature to measure melatonin rhythm's response to bright light therapy and applying this to night shift health care workers, this research stands to save the health care industry billions of dollars and improve the lives of millions of people.

\section{Methodology}

The chosen method used to conduct this research was a case study approach, which allowed for a detailed report of a single individual's reaction to bright light therapy. A quantitative experimental approach would have been preferred because it would allow the treatment groups to be randomized and therefore would have led to a better analysis of the interval and constant treatments. However, after months of recruiting, only one participant was able to be secured. This led to the deviation from the experimental quantitative approach.

This case study consisted of one health care worker taking their own temperature over a three-week interval. The participant had three different sleep schedules: one while working night shift, one while off night shift, and one while transitioning from night shift to non-night shift. While on night shift, the participant woke at 6 p.m. and went to sleep at 10 a.m. and worked from 7 p.m. -7 a.m. While off night shift, the participant woke at 9 a.m. and went to bed at midnight. One day a week the participant transitioned from night shift to non-night shift where they woke at 2 p.m. and went to bed at 11 p.m. The number of days the participant worked night shift varied over the three weeks, however the time frames of all three sleep schedules remained constant throughout the study. During the first week, the participant worked four days of night shift. No bright light therapy was administered, and it therefore acted as a baseline evaluation. Bright light therapy of between 650-750 lux was administered throughout weeks two and three using a Circadian Optics Light Therapy Lamp. This lamp was set on the participants desk within their office, for this was the central location in which they were located while not running rounds. The lamp was set approximately 18 inches from the participant's face. These conditions remained constant throughout weeks two and three, however the time period and duration of exposure differed. The second week consisted of bright light therapy administered constantly during the participant's work shift; this allowed the participant to receive bright light therapy whenever they were in their office, although this specific amount of time was not measured. The participant worked four days of night shift during the second week. The third and final week of treatment consisted of interval bright light therapy. The participant turned on the light box four times for 15 minutes each and separated the intervals by 45 minutes. The first interval would start an hour and 45 minutes into their shift, at 8:45 p.m., and this was be repeated three more times.

Temperature was measured hourly, from the time the participant woke, till the time they went to bed, each day, during the three-week interval. Along with hourly temperatures, the participant also took one temperature 90 minutes prior to their set wake time, each day, in order to measure their temperature minimum. During the three weeks 
of trials, the participant entered all temperature data into an excel data sheet provided to them. With this data, averages were calculated for temperatures taken on and off night shift for each of the three weeks. This data allowed for the participant's temperature curve while on night shift, to be compared to their temperature curve while off night shift, for each of the three weeks as a means to analyze the participant's circadian rhythms. This analysis will reveal whether circadian misalignment occurred during week one, two, and/or three. The averages of temperatures taken on night shift during weeks two and three were also compared to the averages taken during week one in order to compare the shift in temperature curves of week two and three to the temperature curve of week one. This comparison between the shift in temperature curves will reveal differences regarding the effectiveness between each lighting condition.

\section{Results}

After comparing the average temperatures between consecutive weeks, temperature curves were able to be constructed and compared. The average temperatures of week one (Figure 2), while on night shift, are consistent with those of misaligned circadian rhythms.

The participant hit a temperature minimum of 96.13 degrees Fahrenheit at 2 a.m., or hour nine, during week one, while on night shift. Temperature minimums following circadian alignment, should occur at approximately 4:30 p.m. while the participant is working night shift. This curve, however, shows that the temperature minimum is occurring 14.5 hours before its desired time, during the middle of the participants working shift. While off night shift, temperatures during week one follow the trend of aligned circadian rhythms. The participant hits a temperature minimum of 97.27 degrees Fahrenheit at 7:30 a.m., which is 90 minutes prior to the participants habitual wake time.

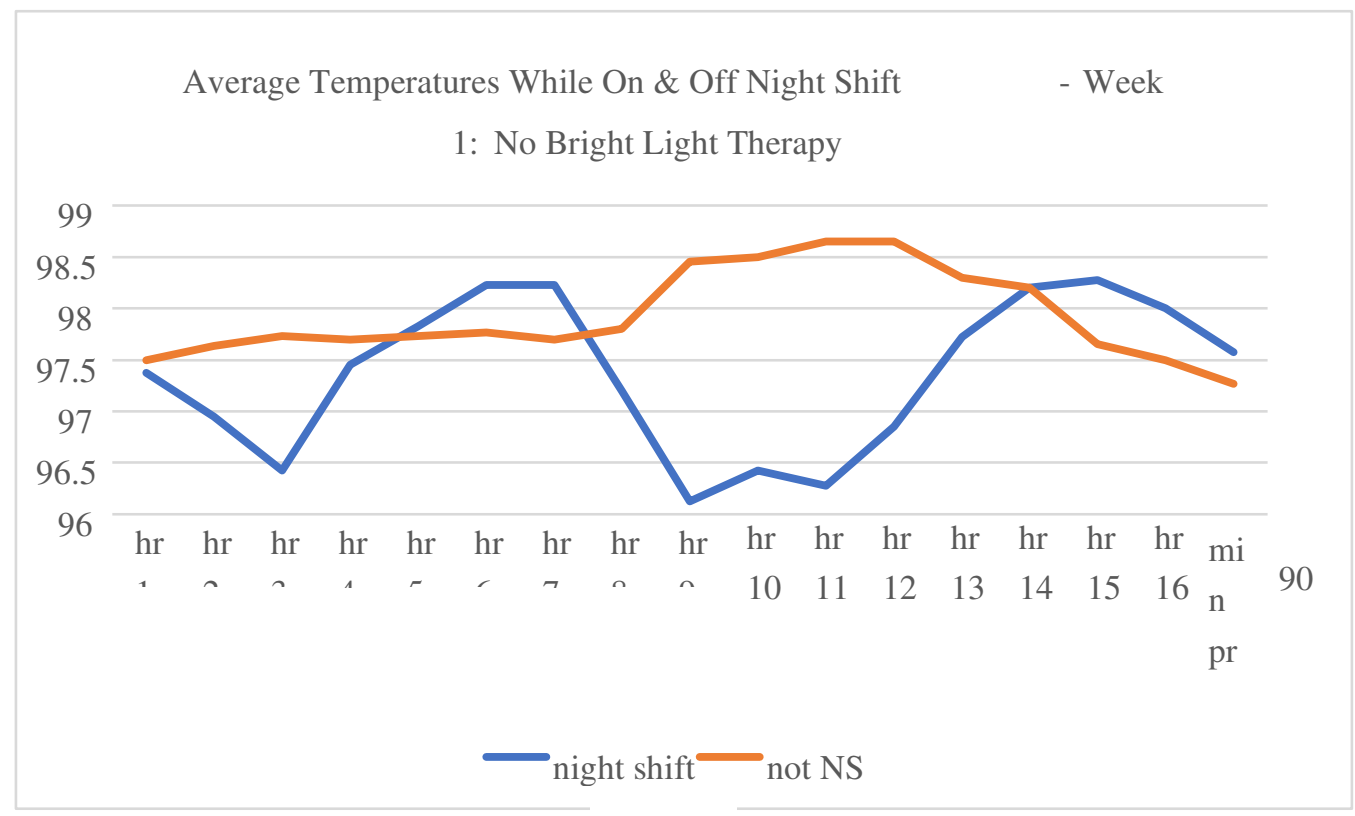

Figure 2

Week two temperatures show only slight circadian misalignment while on night shift (Figure 3). A temperature minimum of 96.65 degrees Fahrenheit occurs 90 minutes prior to the habitual wake time at 4:30 p.m., as it should, during night shift. The curve, however, also drops off at hour four and hit a minimum of 96.68 degrees Fahrenheit at hour eleven. This shows that circadian rhythms are still slightly displaced because an unusually low temperature is being reached during the work shift. Similar to week one, while off night shift during week two, temperature trends follow aligned circadian rhythms. Temperatures slowly increase and reach a peak around hour 10 and then hit a minimum of 96.4 degrees Fahrenheit at 7:30 a.m. 


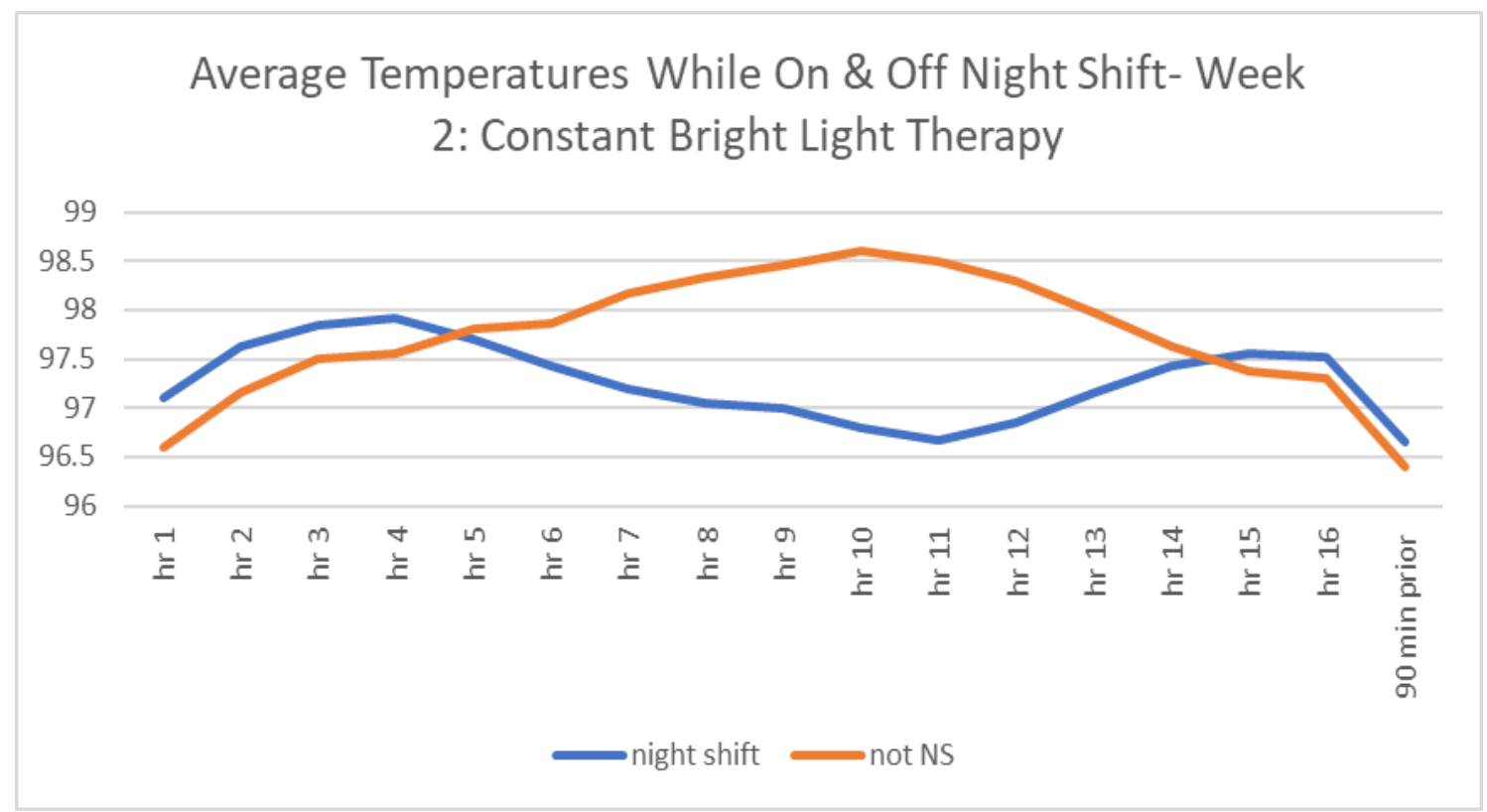

Figure 3

During week three, temperature curves for both on and off night shift show no signs of circadian misalignment (Figure 4). Both curves hit their minimum 90 minutes prior to the participant's habitual wake time. The temperature curve shows a slight increase from the wake time to about hour ten, then a slow decline until the minimum temperature is reached, which is consistent with aligned circadian rhythms.

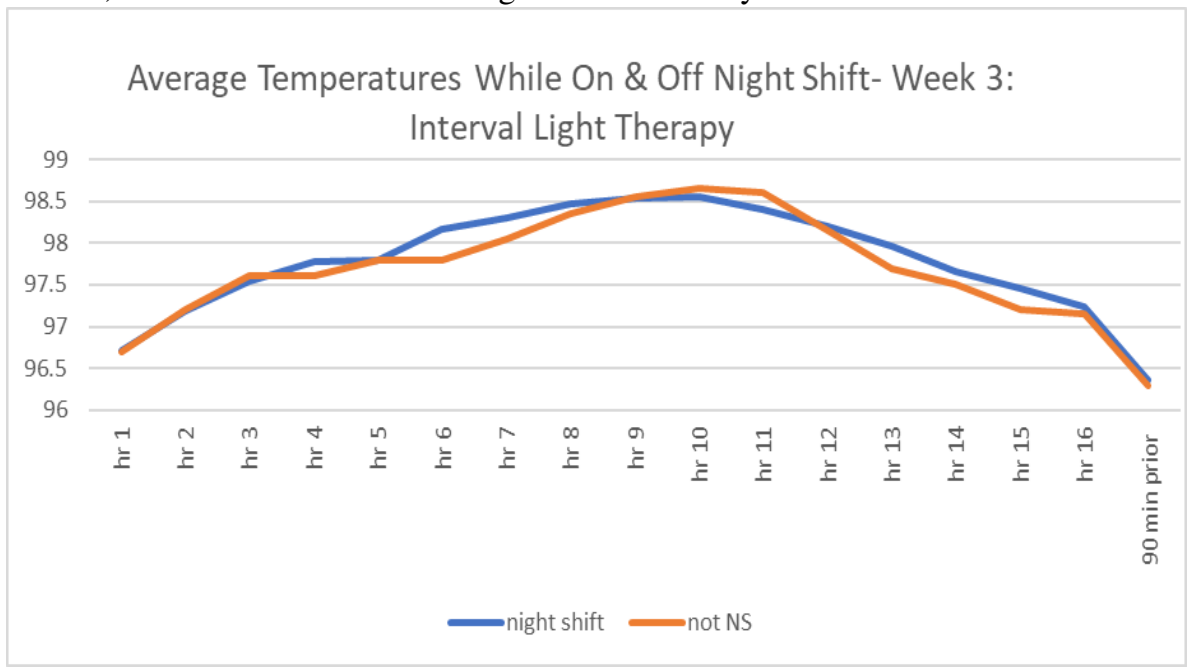

Figure 4

The temperature curves from weeks two and three shift from the temperature curve from week one, which served as a baseline for evaluation since there was no bright light therapy administered. As Figure 5 depicts, the temperature curve for week two shifted slightly from week one and week three's temperature curve shifted greatly from week one. The constant bright light therapy administered in week two caused the temperature curve to shift from a sharp decrease of 2.1 degrees from hour seven till hour nine in week one, to a gradual decrease in temperature of only 1.25 degrees from hour four till hour eleven. This shows a gradual shift to a more constant temperature curve which follows the trend for circadian alignment. The temperature minimum 90 minutes prior to the participants' habitual wake time also shifts from 97.58 during week one, to 96.65 during week two. The interval light therapy during week three, however, caused the greatest shift in temperature curve compared to week one. Week one's temperature 
curve shifted from spiking and dropping erratically, to steadily increasing up until hour ten, and steadily decreasing until hitting its temperature minimum 90 minutes prior to the participant's habitual wake time during week three. This temperature curve achieved in week three is more constant than that from week two.

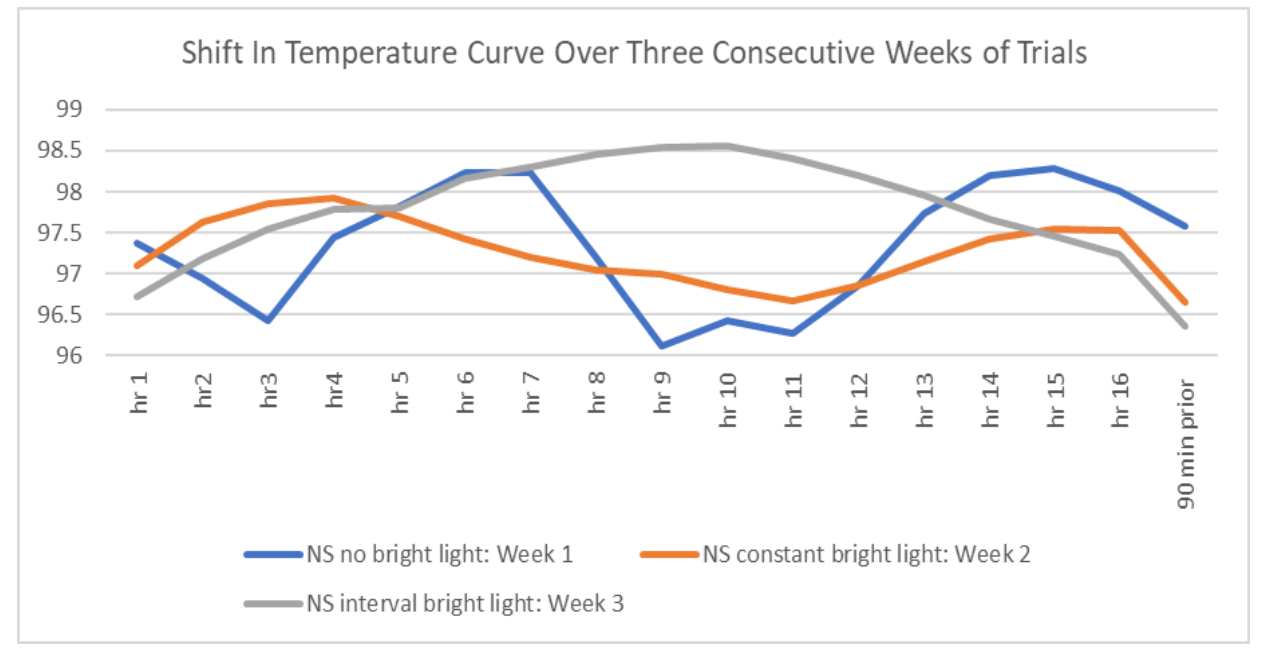

Figure 5

\section{Discussion}

\section{Implications}

This research first exemplifies that circadian misalignment did occur during shift working days and did not occur while off night shift. This conclusion is shown through the comparison of the participant's temperature curve while on night shift, to their temperature curve while off night shift, for each of the three weeks. During week one, the minimum temperature hits 14.5 hours before the desired time and during week two, an unusually low temperature of 96.68 degrees Fahrenheit occurs during the work shift. This trend occurs because the body's circadian clock is not transitioning from day shift, where temperature minimum occurs during the biological night, to night shift where it needs to occur at 4:30pm. Therefore, temperature continues to peak as it would while on a day shift schedule. The body cannot adapt to the light/dark disturbances it experiences while working night shift and therefore circadian misalignment occurs. The comparison of temperature curves while on and off night shift also shows that the participant's circadian rhythms were not disrupted off night shift. This shows that transitioning between shifts did not significantly affect the participant's circadian clock on days off, for their body's temperature curve continued to follow the trend for aligned circadian rhythms while not on shift work. This research, secondly, shows that bright light therapy does cause circadian rhythms to shift. While there was no bright light therapy, temperature minimum occurred during the working shift at hour nine. However, when bright light therapy was administered during weeks two and three, temperature minimums shifted and occurred 90 minutes prior to habitual wake time. The new temperature curves resulting from bright light therapy in weeks two and three follow the trend of circadian misalignment, therefore the researcher accepts the hypothesis that light therapy is an effective way to reduce light induced circadian misalignment.

The shift in temperature curves during weeks two and three from the temperature curve in week one concludes that, not only does generalized bright light therapy shift circadian rhythms, but also that certain factors helped the participant yield a more constant temperature curve. Since the temperature curve for week three stabilized more than the temperature curve from week two, it could be concluded that the interval bright light therapy yielded better results than the constant bright light therapy in week two. This is the conclusion that Smith, Fogg, and Eastman (2009) came to after conducting their interval bright light therapy study. They stated that constant bright light therapy caused a phase shift that was not practical for individuals who had to transition from on and off shift work and hypothesized 
that interval bright light therapy of 4100 lux would achieve a type of phase shift that caused melatonin to peak at a comprisable time between day and night shift. They concluded this after only eight days of night shift work. The interval bright light therapy treatment of week three did yield better results than that of week two, but another possible explanation for this improvement, besides the type of bright light therapy, is the consecutive weeks of exposure. The test results gathered from the study conducted by Shanahan \& Zeitzer (1997) concluded that three weeks of night shift while exposed to bright light of 5,000 lux during the entire night shift yielded melatonin to peak at the proper time, during the participant's sleep episode. The results gathered from the researcher's study during this experiment show that week three of bright light therapy, in which the participant received interval bright light therapy, achieved the most stable temperature curve, but the reason for why is inconclusive. Since the researcher could not sperate the timing between trials due to time constraints, it cannot be concluded whether week three was more effective due to the type of light therapy used or because it was the second consecutive week of bright light therapy treatment.

These results do however lead the researcher to understand that circadian misalignment did not affect the participant on days off night shift, so a comprisable melatonin peak time would be unnecessary. The interval bright light therapy during this study achieved a temperature minimum 90 minutes prior to the participant's habitual wake time while on and off night shift, which does not put the sleepiest circadian time early in the sleep period after night shifts and late in the sleep period on days off, as was stated in Smith, Fogg, and Eastman's study. Therefore, a comprisable phase shift was not needed in this study, as previously described, for the participant did not experience circadian misalignment on days off night shift before bright light therapy, and a comprisable phase shift was not achieved, for temperature minimum continued to peak 90 minutes prior to the participant's habitual wake time after bright light therapy. The results also lead the researcher to the understanding that light intensities of only 650-750 lux can yield a shift in circadian rhythms, with multiple days of exposure.

\section{Limitations}

Potential pitfalls of my research include the lack of subjects and therefore inability to randomize treatment groups. Timing was also a constraint placed on the researcher and limited the researcher's ability to separate the weeks between constant light therapy and interval light therapy, as discussed earlier. A conclusive reason for why week three yielded the best results can therefore not be reached. Laboratory conditions were not available to conduct the study, so outside light influences were not able to be controlled. Temperature measurements could have fluctuated due to this. Results in this study cannot be generalized to larger populations since only one participant was studied. Individuals may react differently to bright light therapy based on age, gender, race, or other factors. How one individual's circadian rhythms shift cannot be generalized to conclude that is how everyone will react.

\section{Future Direction}

This research extends the existing knowledge of melatonin rhythm as a marker for the human circadian timing rhythm. Previous research was furthered by applying this mechanism of melatonin rhythm, defined by the phase response curve, and specifically applying it to health care workers. This study needs to be repeated in the future using a quantitative experimental approach. This will allow the three different treatment groups to be randomized and separated. By separating the three different types of bright light therapy into different treatment groups, a better conclusion can be made to determine whether interval or constant bright light therapy yields better results. A larger sample size will also allow one to see whether light therapy effects other individuals the same as this participant and will lead to more reliable results. By gathering a better understanding of how melatonin responds to bright light therapy, health care workers can incorporate bright light therapy into their work environment. This bright light therapy will help them to better adapt to light/dark disturbances and improve their quality of life. By improving sleep and decreasing fatigue, medical error will be reduced, and patient well-being will improve as a result. The medical industry stands to save billions of dollars globally by instituting methods through which their workers can receive bright light therapy. 


\section{Acknowledgments}

I would like to thank my teacher John Murnan for helping me with this project.

\section{References}

Baron, K. G., \& Reid, K. (2014). International review of psychiatry [Circadian misalignment and health]. International Review of Psychiatry, 26(2), 139-154. https://doi.org/10.3109/09540261.2014.911149

Boyce, P.1,2 Hopwood, M. (2013). Manipulating melatonin in managing mood. Acta Psychiatrica Scandinavica, 128, 16-23. https://doi.org/10.1111/acps.12175

Han-Xue, L. (2019). The role of circadian clock genes in tumors. OncoTargets \& Therapy, 12, 3645-3660. https://doi.org/10.2147/OTT.S203144

Khan, S., Hou, H., Duan, P., \& Yao, L. (2018). Shift work-mediated disruptions of circadian rhythms and sleep homeostasis cause serious health problems. International Journal of Genomics, 1-11. https://doi.org/10.1155/2018/8576890

Lerman, S., Eskin, E., Flower, D., George, E., Gerson, B., Hartenbaum, N., . . Moore-Ede, M. (2012). Fatigue risk management in the workplace. Journal of Occupational \& Environmental Medicine, 54(2), 231-258. https://doi.org/10.1097/JOM.0b013e318247a3b0

Lewy, A. (2010). Clinical implications of the melatonin phase response curve. The Journal of Clinical Endocrinology and Metabolism, 95(7), 3158-3160. https://doi.org/10.1210/jc.2010-1031

Moore-Ede, M., \& Platika, D. (n.d.). Health risks of light at night. A Circadian White Paper, 116. Retrieved from https://circadianlight.com/images/pdfs/White\%20Paper/CL_HealthRiskWhite.pdf

Shanahan, T., \& Zeitzer, J. (1997). Resetting the melatonin rhythm with light in humans. Journal of Biological Rhythms, 12(2), 556-566. Retrieved from Complementary Index database. (Accession No. 9712096420)

Smith, M., Fogg, L., \& Eastman, C. (2009). Practical interventions to promote circadian adaptation to permanent night shift work: Study 4. Journal of Biological Rhythms, 24, 161-172. https://doi.org/10.1177/0748730409332068. 